\title{
Effects of phonetic symbolism on paired-associate learning
}

\author{
RONALD A. COHEN and CHIZUKO IZAWA \\ Tulane University, New Orleans, Louisiana 70118
}

\begin{abstract}
To investigate relations, if any, between phonetic symbolism and learning, a paired-associate learning experiment was conducted with two stimulus variations (complete vs. incomplete figures) and three response variations [repetitive vs. nonrepetitive same (S) vs. nonrepetitive different (D) disyllables, where $\mathrm{S}$ and $\mathrm{D}$ were defined in terms of the first vowel of the repetitive group]. Disyllables produced significant effects in all aspects of data. Effects of figures were complex: First-trial data (approximating word matching) indicated suggestive differences, with significant Figure by Disyllable interactions; but overall performance produced insignificant differences between figures. Both figures and disyllables significantly interacted with learning trials. The advantage of the complete-repetitive combination was observed only early in learning, and that of the incomplete-nonrepetitive combination only late in learning. The second sound of disyllables produced significant effects on learning. Phonetic symbolism research may be facilitated by taking learning processes into account.
\end{abstract}

Ever since Plato, phonetic symbolism, i.e., an intrinsic correspondence between sounds (physical properties) and meanings of words, has been of considerable interest (for reviews see Taylor, 1963, e.g.). Yet, evidence beyond anecdotal or cursory observations in this area has been largely limited to studying individual words: matching words against words or nonsense syllables against nonsense syllables (subjective phonetic symbolism) and examining the lexicon of languages in terms of denotata (objective phonetic symbolism). Recently, however, DeVito and Civikly (1972) approached the issue differently and found repetition of sound within disyllables (e.g., LOLO) is reliably associated wth visual representations of homogeneity (vis-a-vis heterogeneity) and completeness (vis-a-vis incompleteness).

No matter whether phonetic symbolism may be assumed to be universal (e.g., Miron, 1961) or language specific (e.g., Taylor \& Taylor, 1962, 1965), provided that phonetic symbolism is based on language $\mathrm{s}$, such symbolism is likely to be learned, along with the language itself. This is particularly true when phonetic symbolism is assumed to be language specific. Taylor and Taylor (1965), e.g., assumed the identity of subjective and objective phonetic symbolism; the latter, no doubt, involved some sort of learning. On the other hand, phonetic symbolism might be demonstrable with nonsense syllables or foreign words (e.g., Newman, 1933; Sapir, 1929). If so, the learning of phonetic symbolism is minimal. The latter evidence in a sense, however, may lead to an ultimate rejection of the existence

The present study is supported, in part, by a Biomedical Research Grant (69175) from NIH via Tulane University to C. Izawa. The authors are grateful to Janice Rauch for her assistance with data reduction and preparing figures. Requests for reprints should be sent to Chizuko Izawa, Department of Psychology, Tulane University, New Orleans, Louisiana 70118. of phonetic symbolism (defined as intrinsic correspondence between sounds and meanings), since meaning is absent in nonsense syllables or foreign words, in spite of possible meaningfulness or association values.

The present experiment examines relationships, if any, between phonetic symbolism and learning in terms of completeness of visual figures and repetition of sounds found by DeVito and Civikly (1972).

\section{METHOD}

A 2 by 3 factorial design (six conditions) was utilized with two stimulus-term (figure) and three response-term (disyllable) variations, using six 15-pair lists. The stimulus terms consisted of simple geometric figures or common syllables: One type consisted of complete line drawings, whereas another consisted of incomplete drawings with a portion of each figure missing; the missing part occurred either on the right or the left of the figure (about equally often among the figures). The response terms, four-letter disyllables with no apparent meaning, were varied three ways: repetitive (repeat the same sound as NENE, e.g.) and two types of nonrepetitive groups, with one type using the same sound in its first syllable as the repetitive group (as NENA, e.g.) and the other type using two entirely different sounds (as NANO, e.g.). One set of 15 consonants was used throughout all three response-term variations. The conditions were: (1) complete-repetitive, (2) complete-nonrepetitive (S), (3) complete-nonrepetitive (D), (4) incomplete-repetitive, (5) incomplete-nonrepetitive (S), and (6) incomplete-nonrepetitive (D), where $S$ and D represent same and different vowels, respectively.

Under the study(reinforcement)-test method, learning progressed until the 12th study and test cycles were completed. Item presentation order was randomized from cycle to cycle. The subject pronounced each response term aloud on study cycles and responded orally on test cycles. The presentation rates for both study and test events were $3 \mathrm{sec}$ each, with 30 -sec intercycle intervals. Prior to the main task, all subjects had a practice task with six pairs, each representing one of the six conditions, for three study and test cycles, respectively. Sixty introductory psychology students participated, 10 in each condition. Using a semi-Latin square method, the subjects were assigned to one of the six conditions in the order of their appearance. 


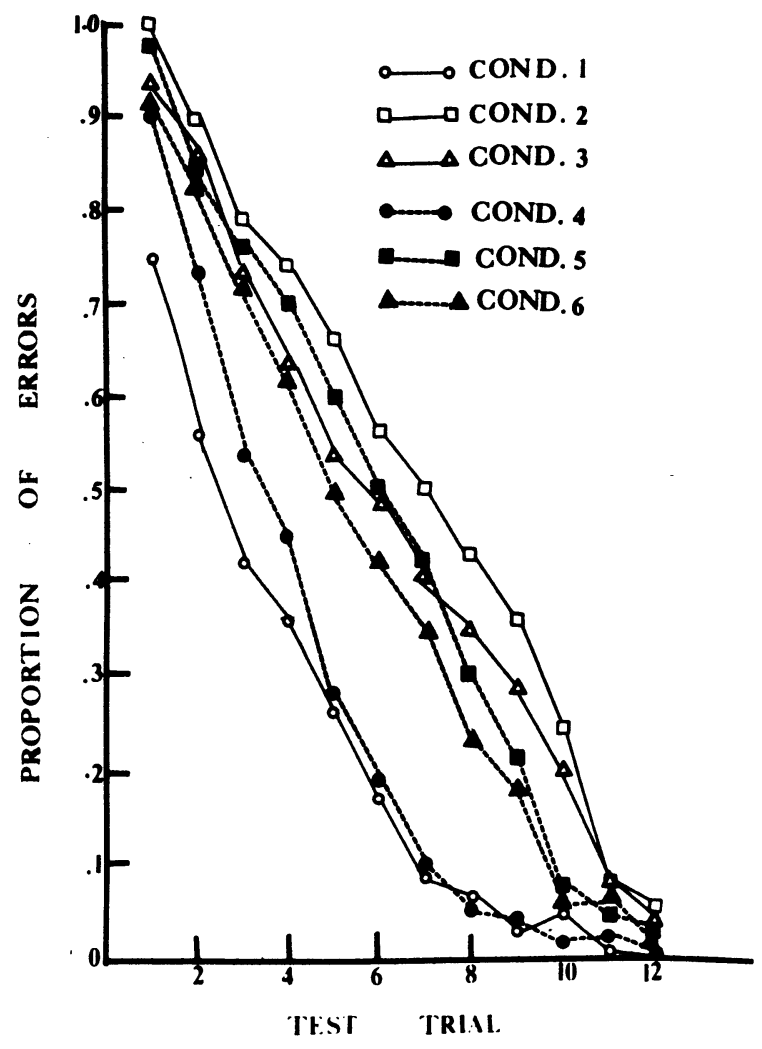

Figure 1. Performance on all conditions in terms of proportion of incorrect responses.

\section{RESULTS AND DISCUSSION}

Tested by the practice task performances, there were no differences in subject variables among the six groups, $\mathrm{F}<1$. Major results are shown in Figure 1. Data analyses were, first, done for overall performances. An analysis in terms of the total errors per subject showed highly significant differences among response terms (disyllables) $[F(2,54)=45.31, p<.001]$. Further analyses indicated a major contributor to the differences to be the great advantage of the repetitive over the nonrepetitive conditions ( $\mathrm{S}$ and $\mathrm{D}$ groups pooled) $[\mathrm{F}(1,56)=83.44$, $\mathrm{p}<.001]$. Differences between the stimulus terms (figures), however, did not reach a significant level $[F(1,54)=1.40, p>.05]$. The smallness of differences was, in part, the result of suggestive interactions between figures and disyllables $[\mathrm{F}(2,54)=2.61, \mathrm{p} \doteq .08]$.

An analysis of acquisition trials showed that both interactions between figures (stimuli) and trials and interactions between disyllables (responses) and trials were highly significant $[\mathrm{F}(11,594)=4.57$ and $\mathrm{F}(22,594)=10.12$, respectively, with $\mathrm{p}<.001$ ] . Complex differences according to learning stages are shown in Figure 2, with each disyllable group separated. Quite intriguingly, when response disyllables consisted of repetitive sounds (e.g., NENE, Figure 2, left panel), although differences were minimal toward the latter half of acquisition, Condition 1 with complete figures (stimulus terms) was more advantageous than Condition 4 with incomplete figures during the first four test cycles.
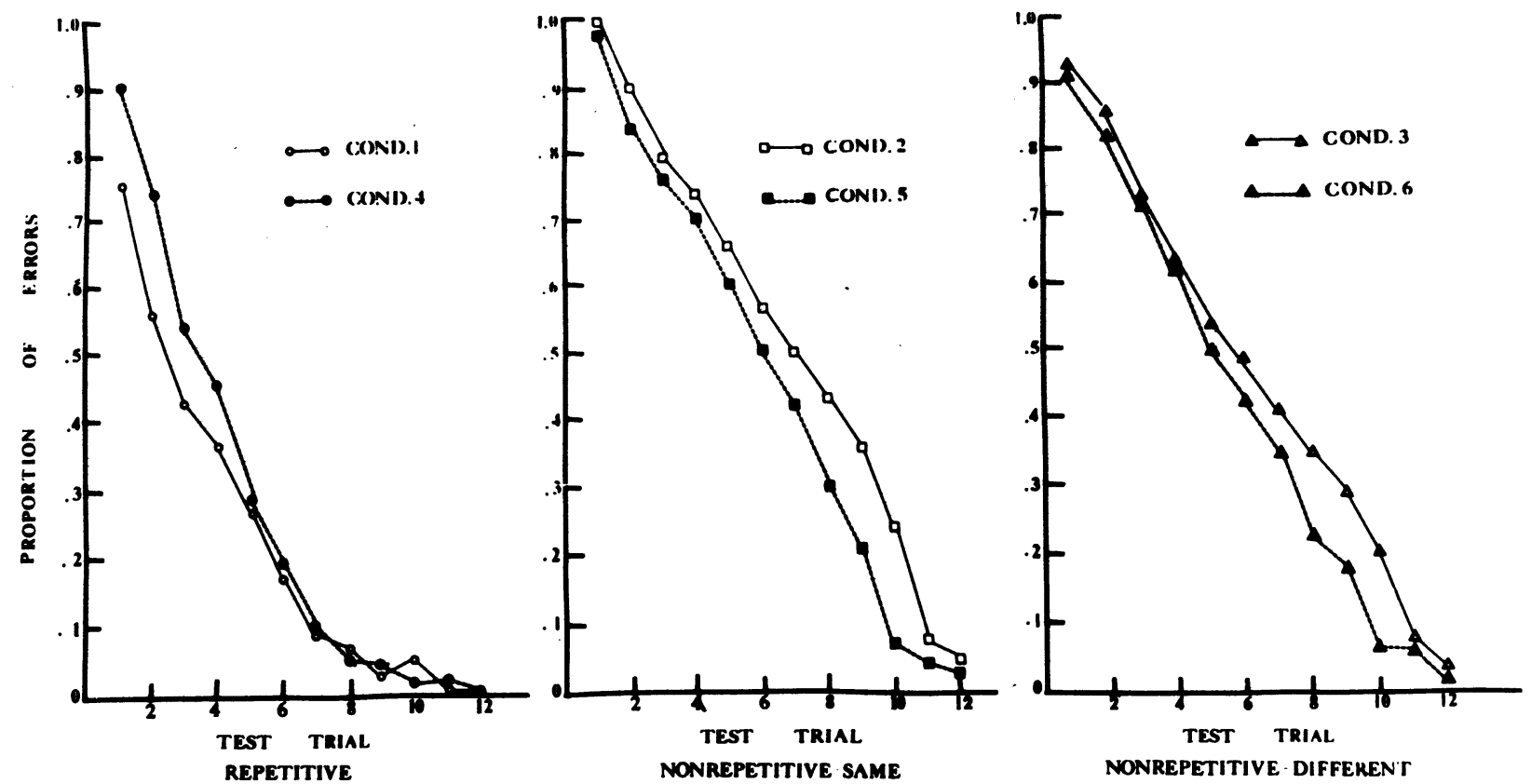

Figure 2. Performance between two stimulus types (complete vs. incomplete figures) for each of the response (disyllable) variations: repetitive (left), nonrepetitive (S) (center), and nonrepetitive (D) (right). 
$[F(1,18)=4.65, p<.05]$. The opposite occurred when nonrepetitive sounds were used for response disyllables (center and right panels). Here, learning was slightly better for incomplete figures paired with nonrepetitive disyllables; although differences were small overall, they reached a significant level in late acquisition trials (6-10) $[\mathrm{F}(1,18)=5.79, \mathrm{p}<.05$ between Conditions 2 and $5 ; \mathrm{F}(1,18)=4.10, \mathrm{p} \doteq .05$ between Conditions 3 and 6].

Phonetic symbolism research typically includes some aspects of matching words, nonsense syllables, or figures, usually in one trial. Closest to such response measures in the present study are the first-trial performances. To allow some comparisons with previous studies, data on the first trial of all six conditions were analyzed: Again, highly significant differences among disyllables (response terms) were shown $[\mathrm{F}(2,54)=18.86, \mathrm{p}<.001]$. Quite interestingly, differences between figures (stimuli) were large enough to suggest trends $[F(1,54)=3.28, .05<p$ $<.10]$, and interactions between stimulus and response terms were significant $[F(2,54)=6.61, p<.01]$. Essentially the same results were obtained when the two types of nonrepetitive disyllables (pooled) were contrasted with the repetitive ones [significant disyllable differences, $F(1,56)=31.94, p<.001$, and interactions, $F(1,56)=12.66, \quad p<.001$, as well as suggestive differences between figures, $F(1,56)=3.15, .05<$ $p<.10]$. The results from the first trials are more or less in agreement with findings by DeVito and Civikly (1972). A reason why figures did not generate significant differences may be that they were used as stimulus terms, whose effects are less strong than response terms.

However, when all learning stages were considered, deviations from DeVito and Civikly's (1972) data emerged. As seen in Figure 2, the advantage of the complete figures paired with repetitive disyllables early in learning is indeed in accord with DeVito and Civikly (1972), but the advantage did not hold during the latter half of learning stages. Conversely, the expected supremacy of the incomplete figure/nonrepetitive disyllables combinations was not present in early learning (Figure 2, center and right panels); rather, it surfaced relatively late in learning.

Perhaps the most prominent sound of words that control phonetic symbolism related to the initial sound and the first vowel together with the stressed vowel (e.g., Taylor \& Taylor, 1962, 1965). In the present study, the response terms of Conditions 1 and 2 and Conditions 4 and 5 , respectively, shared both initial consonants and vowels, as well as the same types of figures (stimuli), e.g., NENE vs. NENA for repetitive vs. nonrepetitive (S). When relevant data (Conditions 1, 2, 4, and 5) were analyzed, there were significant differences between repetitive and nonrepetitive $(S)$ disyllables $[F(1,36)=$ $75.88, \mathrm{p}<.001]$, quite contrary to the expectation from Taylor and Taylor $(1962,1965)$. Although dif- ferences between figures (stimuli) were insignificant, interactions (Figure by Disyllable) were significant $[\mathrm{F}(1,36)=4.51, \mathrm{p}<05]$. Interestingly, when the initial trials (learning is minimal, approximating wordmatching data) of the above four conditions were considered, not only disyllable differences $[\mathrm{F}(1,36)=35.96$, $\mathrm{p}<.001]$ and interactions $[\mathrm{F}(1,36)=9.36, \mathrm{p}<.01]$ but also figures generated significant differences $[F(1,36)=$ $6.60, \mathrm{p}<.05]$. Apparently, the second sound of disyllables, as well as the first, produced significant roles in the present study.

The present findings of the complex effects of learning stages cannot be adequately explained by current theories, if phonetic symbolism is assumed to be learned, as is tacitly done by Taylor and Taylor (1965), for example. Suppose that the phenomena found by DeVito and Civikly (1972) are learned; then, the advantage expected for complete(figure)-repetitive(sound) and incomplete-nonrepetitive combinations is likely to be more prominent toward the end of learning. The trend was supported by one combination, but the opposite was true for another (Figure 2). Granting that learning of phonetic symbolism and of paired associates may involve different processes, it is still possible that there are processes that underlie phonetic symbolism which may not be readily demonstrable from word-matching techniques alone.

On the other hand, some phonetic symbolism seems to exist with nonsense syllables or foreign words (e.g., Dagiri, 1958; Newman, 1933; Sapir, 1929). If so, little or only minimal learning may be involved in phonetic symbolism. However, in a sense, the very fact that the phenomena exist with nonsense syllables or foreign words may ultimately lead one to reject phonetic symbolism (cf. Bentley \& Varon, 1933; Maltzman, Morrisett, \& Brooks, 1956, e.g., denied the existence of phonetic symbolism). This follows from the fact that both nonsense syllables and foreign words (unknown to the subject) lack meaning. To call blind "guess" or "feeling" or the like "meaning" is debatable. It may be possible to redefine "meaning" as an "intrinsic meaning" of a word independent of the subject's knowledge of it. Such redefinition may be possible with foreign words, but not with nonsense syllables that have no meaning in any language.

The present study suggests that better theory constructions in this field may be facilitated by taking learning aspects of phonetic symbolism into account to a greater extent.

\section{REFERENCES}

Bentley, M., \& Varon, E. An accessary study of "phonetic symbolism." American Journal of Psychology, 1933, 45, 76-86.

DAGIRI, O. On phonetic symbolism. In Y. Endo, K. Hatano, H. Kobayashi, M. Koshimizu, O. Miyagi, H. Nakajima, 
\& T. Obonai (Eds.). Science of language (Vol. 3). Tokyo: Nakayama Shoten, 1958.

DEViro. J. A., \& CivikiY, J. M. Some semantics of repetition: An experiment in phonetic symbolism. Journal of Communication, 1972, 22, 39-47.

Maltzman, I., Morrisett, L., JR., \& Brooks, L. O. An investigation of phonetic symbolism. Journal of Abnormal and Social Psychology, 1956, 53, 249-251.

Miron. M. A cross-linguistic investigation of phonetic symbolism. Journal of Abnormal and Social Psychology, 1961, 62, 623-630.

Newman, S. Further experiments in phonetic symbolism. American Journal of Psychology, 1933, 45, 53-75.
SAPIR, E. A study in phonetic symbolism. Journal of Experimental Psychology, 1929, 12, 225-239.

TAYlor, I. K. Phonetic symbolism re-examined. Psychological Bulletin, 1963, 60, 200-209.

TAYLOR, I. K., \& TAYLOR, M. M. Phonetic symbolism in four unrelated languages. Canadian Journal of Psychology, 1962, 16, 344-356.

Taylor, I. K., \& TAYlor, M. M. Another look at phonetic symbolism. Psychological Bulletin, 1965, 64, 413-427.

(Received for publication June 28, 1976.) 